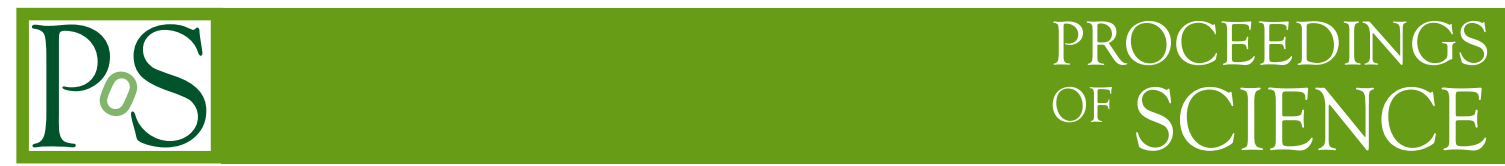

\title{
Fermion Bag Solutions to Sign Problems
}

\section{Shailesh Chandrasekharan*}

E-mail: sch@phy.duke.edu

The fermion bag approach provides new solutions to sign problems. Here we show this by using a simple example of a lattice Yukawa model constructed with staggered fermions and containing a $Z_{2}$ chiral symmetry. We argue that in the conventional formulation of the model the fermion determinant is real but not necessarily positive. However, when formulated in terms of fermion bags, the sign problem is absent. The solution requires the fermionic part to be formulated in terms of fermion bags, while the bosonic part needs to be reformulated in world-line variables.

The 30th International Symposium on Lattice Field Theory

June 24 - 29, 2012

Cairns, Australia

\footnotetext{
* Speaker.
} 


\section{Introduction}

Lattice field theories containing fermions often suffer from sign problems in three or more space-time dimensions and solving them continues to be an important challenge. Famous examples where sign problems have hindered progress, include lattice QCD in the presence of a baryon chemical potential and Fermi liquids with purely repulsive interactions. These sign problems are considered very difficult and any progress in solving them will be a break through. Interestingly, many simpler lattice field theories including four-fermion models and Yukawa models also suffer from sign problems in the conventional formulations and in order to solve them it is necessary to double the fermion degrees of freedom [1,2]. Recently a new approach to lattice field theories has emerged in which partition functions are written in the world-line representation [3]. Fermion sign problems are then solved by summing over the world-lines in regions of space-time refered to as fermion bags [4]. In this new approach, some sign problems, that are present in the conventional approach, can be solved $[5,6]$. Using a simple example of a $Z_{2}$ symmetric Yukawa model, here we show how the new fermion bag approach solves some unsolved sign problems.

\section{The Sign Problem}

Let us consider a simple lattice Yukawa model in which staggered fermions interact with an Ising field. The action of the model is given by

$$
S_{s}=\sum_{x, y} \bar{\psi}_{x}\left(D^{s}[\sigma]\right)_{x y} \psi_{y}+S_{b}[\sigma]
$$

where $\bar{\psi}_{x}, \psi_{x}$ are Grassmann valued fields on the lattice site $x$ with $V$ sites. The Ising field $\sigma_{x}= \pm 1$ is governed by the action,

$$
S_{b}[\sigma]=-\beta \sum_{\langle x y\rangle} \sigma_{x} \sigma_{y} .
$$

Here $\langle x y\rangle$ refers to nearest neighbor sites. The matrix $D^{s}[\sigma]$ is the $V \times V$ Dirac operator whose matrix elements are given by

$$
\left(D^{s}[\sigma]\right)_{x y}=-g \sigma_{x} \delta_{x, y}+\left(D^{s_{0}}\right)_{x y}, \quad\left(D^{s_{0}}\right)_{x y}=\sum_{\alpha} \eta_{\alpha, x} \nabla_{x y}^{\alpha},
$$

where $D^{s_{0}}$ is the massless staggered Dirac matrix and the fluctuating mass term depends on the Ising field. The constants $\beta$ and $g$ are the couplings of the model which are assumed to be positive. The index $\alpha$ represents direction, $\eta_{\alpha, x}$ are the staggered fermion phase factors and

$$
\nabla_{x y}^{\alpha}=\frac{1}{2}\left(\delta_{x, y+\hat{\alpha}}-\delta_{x+\hat{\alpha}, y}\right) .
$$

It is easy to verify that the action in (2.1) is invariant under the following $S U(2)$ transformations :

$$
\left(\begin{array}{c}
\psi_{x} \\
\bar{\psi}_{x}
\end{array}\right) \rightarrow V\left(\begin{array}{c}
\psi_{x} \\
\bar{\psi}_{x}
\end{array}\right) \text { at even sites, }\left(\bar{\psi}_{x} \psi_{x}\right) \rightarrow\left(\bar{\psi}_{x} \psi_{x}\right) V^{\dagger} \text { at odd sites }
$$

where $V \in S U(2)$ is part of the flavor symmetry and has been recently used in [7]. In addition the action is also invariant under the following $Z_{2}$ chiral symmetry: $\psi_{x} \rightarrow i \varepsilon_{x} \psi_{x}, \bar{\psi}_{x} \rightarrow i \varepsilon_{x} \bar{\psi}_{x}$ and 
$\sigma_{x} \rightarrow-\sigma_{x}$ where $\varepsilon_{x}= \pm 1$ is the site parity. At small values of $\beta$, when the Ising field describes massive particles, one can imagine integrating over the $\sigma$ field and the theory is equivalent to a four-fermion model.

In the conventional approach, after integration over the Grassmann variables, the partition function of the model is given by

$$
Z=\sum_{[\sigma]} \mathrm{e}^{S_{b}[\sigma]} \operatorname{Det}\left(D^{s}[\sigma]\right)
$$

Due to the fluctuating mass term, $\operatorname{Det}\left(D^{s}[\sigma]\right)$ can only be guaranteed to be real but not positive. This is the origin of the sign problem in the conventional approach. In the symmetry broken phase where $\sigma_{x} \approx \sigma_{0}+\eta_{x}$, as long as $\eta_{x} \ll \sigma_{0}$ the sign problem can be expected to be mild or absent. However, close to critical points where $\sigma_{0} \rightarrow 0$, the sign problem can in principle become severe. We will show below that it is possible to reformulate the partition function using world-line and fermion bag variables such that the sign problem is absent.

\section{Fermion Bag Approach}

Instead of intergrating over all the fermion degrees of freedom, the idea behind the fermion bag approach is to collect fermion degrees of freedom into groups so that integrating over each group produces positive answers. While this is not always possible, we have found that the approach does solve at least some sign problems. The sign problem in the $Z_{2}$ Yukawa model discussed in the above section is one example where the fermion bag approach succeeds in solving the sign problem completely.

We begin with the partition function for the action in (2.1), which is given by

$$
Z=\sum_{[\sigma]}\left(\prod_{\langle x y\rangle} \mathrm{e}^{\beta \sigma_{x} \sigma_{y}}\right) \int[d \bar{\psi} d \psi] \mathrm{e}^{-\sum_{x y} \bar{\psi}_{x}\left(D^{s 0}\right)_{x y} \psi_{y}} \prod_{x} \mathrm{e}^{g \sigma_{x} \bar{\psi}_{x} \psi_{x}}
$$

We then expand

$$
\mathrm{e}^{g \sigma_{x} \bar{\psi}_{x} \psi_{x}}=1+g \sigma_{x} \bar{\psi}_{x} \psi_{x}=\sum_{n_{x}=0,1}\left(g \sigma_{x} \bar{\psi}_{x} \psi_{x}\right)^{n_{x}}
$$

where in the last step we have introduced a discrete monomer field $[n]$. If $n_{x}=1$ then the site contains a monomer, otherwise the site is considered empty. In terms of this monomer field it is easy to write the partition function as

$$
\begin{aligned}
Z=\sum_{[n]}\left\{\sum_{[\sigma]\langle x y\rangle} \mathrm{e}^{\beta \sigma_{x} \sigma_{y}} \sigma_{z_{1}} \sigma_{z_{2}} \ldots \sigma_{z_{k}}\right\} \times \\
\quad\left[\int[d \bar{\psi} d \psi] \mathrm{e}^{-\sum_{x y} \bar{\psi}_{x}\left(D^{s_{0}}\right)_{x y} \psi_{y}} g \bar{\psi}_{z_{1}} \psi_{z_{1}} g \bar{\psi}_{z_{2}} \psi_{z_{2}} \ldots g \bar{\psi}_{z_{k}} \psi_{z_{k}}\right]
\end{aligned}
$$

where we assume the monomer configuration $[n]$ contains $k$ monomers located at the sites $z_{1}, z_{2}, \ldots z_{k}$. Let us refer to the term in the curly bracket as the bosonic part and the term in the square bracket as the fermion part.

Let us first focus on the fermionic part which is a Grassmann integral. We first note the standard identities :

$$
\int d \bar{\psi}_{x} d \psi_{x} \bar{\psi}_{x} \psi_{x}=-1, \quad \int[\bar{\psi} \psi] \mathrm{e}^{-\sum_{x y} \bar{\psi}_{x} M_{x y} \psi_{y}}=\operatorname{Det}(M)
$$




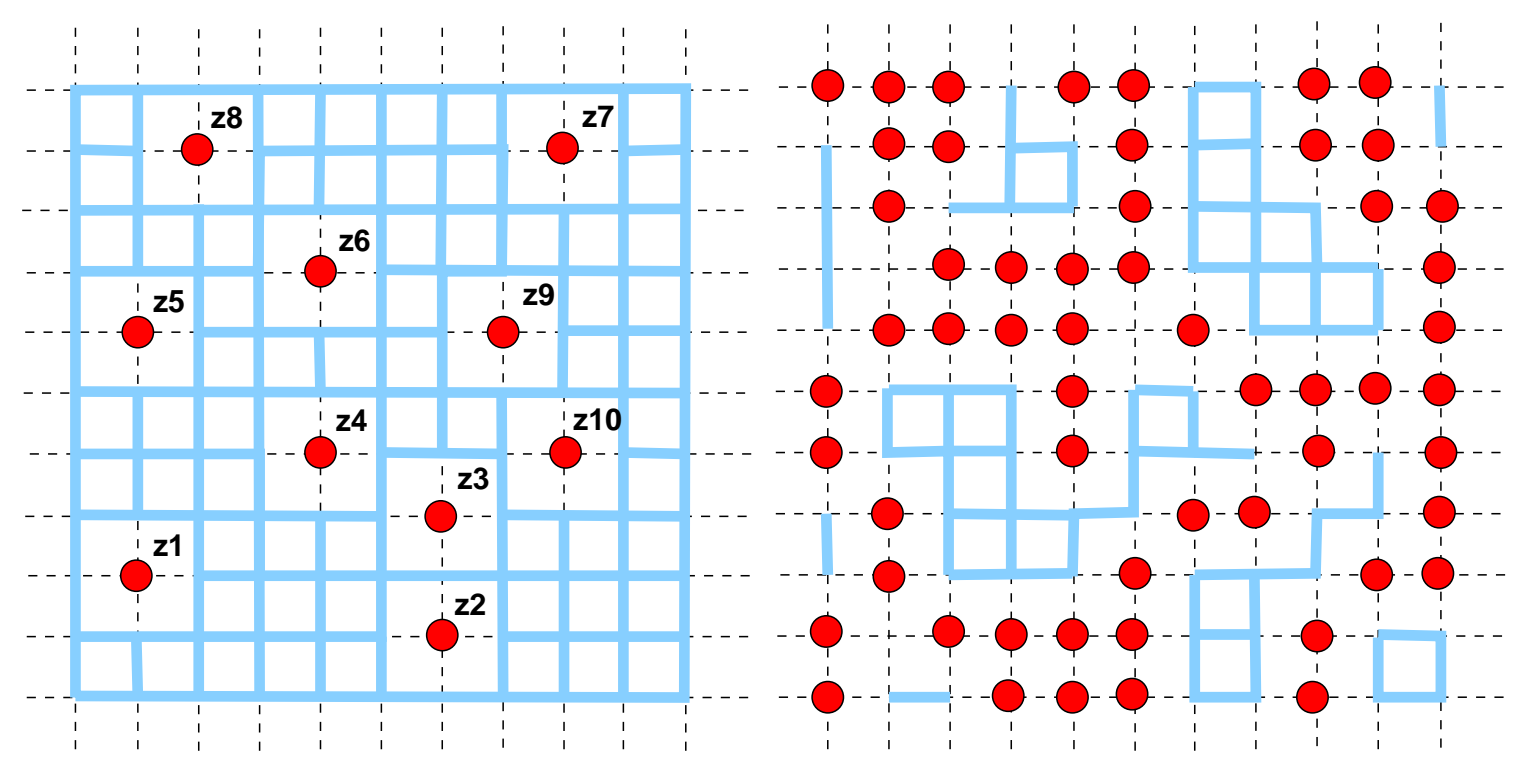

Figure 1: The left figure illustrates a fermion bag configuration in two dimensions with ten monomers. Thus the configuration contains 11 fermion bags, 10 of which are monomers with weight $(-g)$ each and one large free fermion bag with weight $\operatorname{Det}\left(W^{s_{0}}\right)$ where $W^{s_{0}}$ is the free staggered Dirac matrix but on a lattice which does not contain the ten monomers. The right figure illustrates how the single large free fermion bag splits into many smaller bags as the number of monomers increase.

Using these the full fermionic integral can be performed in two steps. First we integrate over Grassmann variables associated with the monomer sites $z_{1}, z_{2}, \ldots z_{k}$. Since the sources already contain both $\bar{\psi}$ and $\psi$ at each of these sites, we can simply set to zero the terms in the exponent containing these sites. Let $W^{s_{0}}[n]$ be the $(V-k) \times(V-k)$ matrix obtained from $D^{s_{0}}$ by dropping the rows and columns corresponding to $z_{1}, z_{2}, \ldots z_{k}$. The Grassmann integral on each of the $k$ monomer sites gives a -1 , while the integral over the remaining Grassmann variables yields $\operatorname{Det}\left(W^{s_{0}}[n]\right)$. Mathematically this means

$$
\begin{aligned}
{\left[\int[d \bar{\psi} d \psi] \mathrm{e}^{-\sum_{x y} \bar{\psi}_{x}\left(D^{s_{0}}\right)_{x y} \psi_{y}}\right.} & \left.g \bar{\psi}_{z_{1}} \psi_{z_{1}} g \bar{\psi}_{z_{2}} \psi_{z_{2}} \ldots g \bar{\psi}_{z_{k}} \psi_{z_{k}}\right] \\
= & (-g)^{k} \int[d \bar{\psi} d \psi] \mathrm{e}^{-\sum_{x y} \bar{\psi}_{x}\left(W^{s_{0}}[n]\right)_{x y} \psi_{y}}=g^{k} \operatorname{Det}\left(W^{s_{0}}[n]\right) \geq 0 .
\end{aligned}
$$

In the last step we have set $(-1)^{k}=1$ since $\operatorname{Det}\left(W^{s_{0}}[n]\right) \neq 0$ only if $k$ is even. Since $W^{s_{0}}[n]$ is the same as the free staggered fermion matrix, its determinant is positive.

In the above approach we divided the Grassmann variables into those associated with monomers and those associated with the remaining free sites. Thus, every monomer and all the remaining free sites together form fermion bags. The integral over the Grassmann variables within each fermion bag is called the weight of the fermion bag. The weight of each monomer is $-g$ and the weight of the free fermion bag containing all the remaining sites is $\operatorname{Det}\left(W^{s_{0}}[n]\right)$. Since the number of monomers is always guaranteed to be even the product of all the fermion bag weights is positive. When $g$ is large, then the single large fermion bag containing free sites can split into smaller bags (see figure 1). As pointed out in [8,9], we can also use Wick's theorem to show that

$$
\operatorname{Det}\left(W^{s_{0}}[n]\right)=\operatorname{Det}\left(D^{s_{0}}\right) \operatorname{Det}\left(G^{s_{0}}[n]\right)
$$




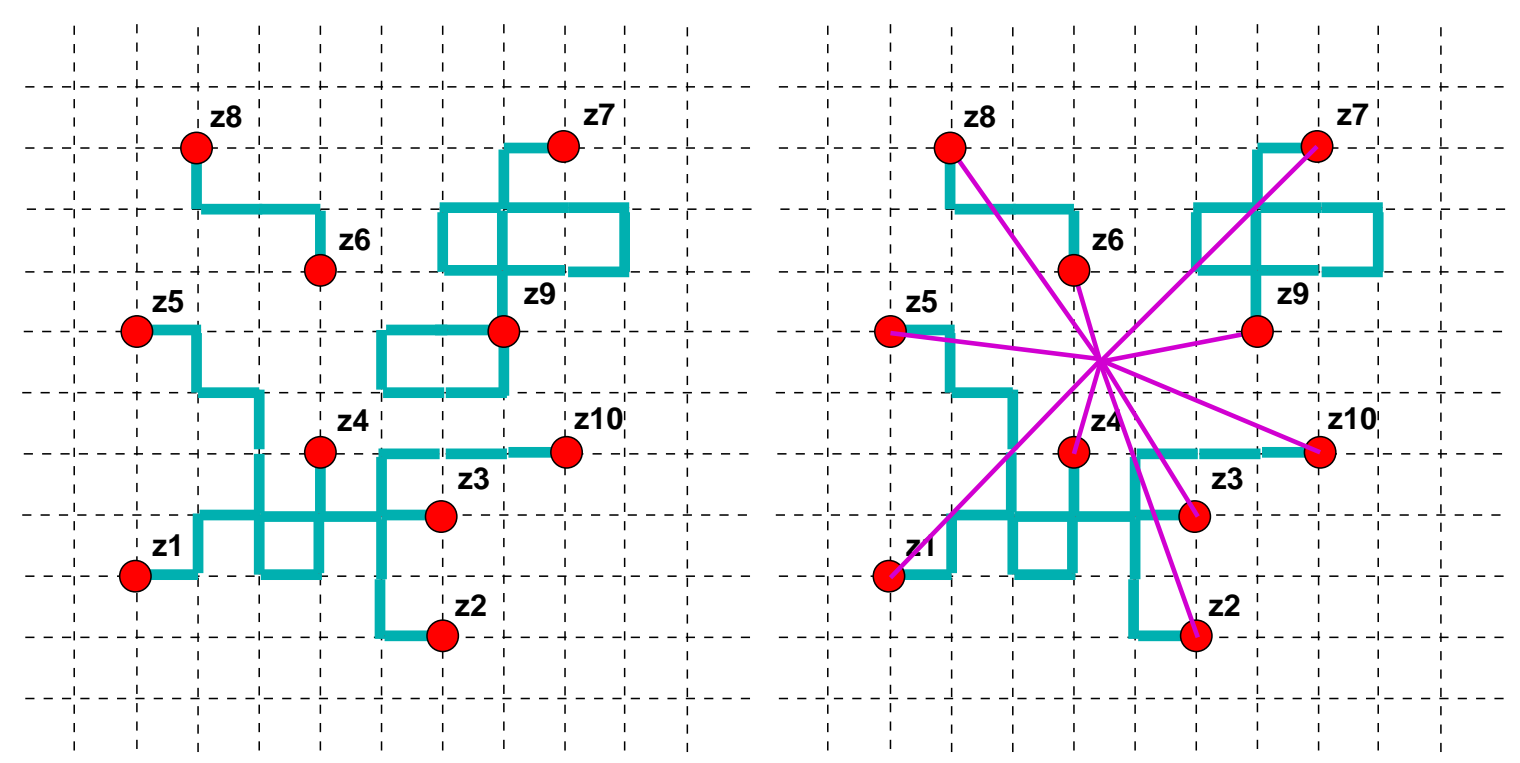

Figure 2: The left figure illustrates the world-line configuration of bonds and monomers. Each site must contain an even number of bonds if it does not have a monomer on it, otherwise it must contain an odd number of bonds. In the right figure we connect all the monomers together to represent a dual fermion bag whose weight is $g^{k} \operatorname{Det}\left(G^{s_{0}}[n]\right)$ and show it along with the world-line configuration of the left figure. Configurations of this type contribute to the parition function.

where $G^{s_{0}}$ is a $k \times k$ free propagator matrix for fermions hopping between the monomers. Equation (3.6) is a duality relation and from the dual view point, the set of monomers with fermions propagating freely through them behaves as a dual fermion bag with weight $g^{k} \operatorname{Det}\left(G^{s_{0}}[n]\right)$. This is shown in the right figure in figure 2.

Having shown that the fermionic integral is positive, next we focus our attention to the bosonic term in (3.3) which is clearly not positive as it stands. Using the identity

$$
\mathrm{e}^{\beta \sigma_{x} \sigma_{y}}=\cosh (\beta)\left[1+\tanh (\beta) \sigma_{x} \sigma_{y}\right]=\cosh (\beta) \sum_{b_{\langle x y}=0,1}\left(\tanh (\beta) \sigma_{x} \sigma_{y}\right)^{b_{\langle x y\rangle}}
$$

on each bond, we introduce a discrete dimer field $[b]$ where a bond contains a dimer if $b_{\langle x y\rangle}=1$. We can then rewrite

$$
\sum_{[\sigma]\langle x y\rangle} \prod^{\beta \sigma_{x} \sigma_{y}} \sigma_{z_{1}} \sigma_{z_{2}} \ldots \sigma_{z_{k}}=(\cosh (\beta))^{d V} \sum_{[b]} \prod_{\langle x y\rangle}(\tanh (\beta))^{\left.b_{\langle x y\rangle}\right\rangle}\left\{\sum_{[\sigma]} \prod_{x}\left(\sigma_{x}\right)^{\gamma_{x}}\right\}
$$

where $\gamma_{x}$ is the total number dimers that touch the site $x$ plus the number of monomers on that site. The sum over the Ising field can be performed using the relation

$$
\sum_{\sigma_{x}}\left(\sigma_{x}\right)_{x}^{\gamma}=2 \delta_{\gamma_{x}, \text { even }}
$$

which says that only dimer configurations that contain an even $\gamma_{x}$ at each lattice site contribute to the path integral. Thus, we see that the bosonic part can be rewritten as

$$
\sum_{[\sigma]} \prod_{\langle x y\rangle} \mathrm{e}^{\beta \sigma_{x} \sigma_{y}} \sigma_{z_{1}} \sigma_{z_{2}} \ldots \sigma_{z_{k}}=2^{V}(\cosh (\beta))^{d V} \sum_{[b]} \prod_{\langle x y\rangle}(\tanh (\beta))^{\left.b_{\langle x y}\right\rangle} \prod_{x} \delta_{\gamma_{x}, \text { even }}
$$


which contains only positive terms.

Thus, substituting the results of (3.5), (3.6) and (3.10) into (3.3) we can write the partition function as a sum over confgurations of monomers and dimers $[b, n]$ such that

$$
Z=2^{V}(\cosh (\beta))^{d V} \operatorname{Det}\left(D^{s_{0}}\right) \sum_{[b, n]} g^{k}\left(\prod_{\langle x y\rangle}(\tanh (\beta))^{\left.b_{\langle x y}\right\rangle} \prod_{x} \delta_{\gamma_{x}, \text { even }}\right) \operatorname{Det}\left(G^{s_{0}}[n]\right)
$$

which is free of sign problems and can be used to design Monte Carlo algorithms.

\section{Discussion}

Using a simple model we have shown that some sign problems that seem unsolvable in the conventional approach can be completely solved in the fermion bag approach. Some sign problems in more complex models with continuous symmetries and those involving Wilson fermions can also be solved using similar ideas [6]. In particular we can study four-fermion models of QCD with interesting chiral symmetries.

A class of Yukawa models where the new solution is applicable can be characterized by the action

$$
S=\sum_{x y}\left\{\bar{\psi}_{x} D_{x y} \psi_{y}+\bar{\chi}_{x}\left(D^{\dagger}\right)_{x y} \chi_{y}\right\}-\sum_{x}\left(g_{1} \phi_{x} \bar{\psi}_{x} \chi-g_{2} \varphi_{x}^{*} \bar{\chi}_{x} \psi_{x}\right)+S_{b}(\phi, \varphi)
$$

where $\bar{\psi}, \psi, \bar{\chi}, \chi$ are independent Grassmann fields, $\phi, \varphi$ are complex boson fields, $g_{1}, g_{2}$ are real positive couplings. Further, the bosonic action $S_{b}(\phi, \varphi)$ has the property that any $2 k$-point correlation function

$$
\int[d \phi d \varphi] \mathrm{e}^{-S_{b}} \phi_{x_{1}} \ldots \phi_{x_{k}} \varphi_{y_{1}}^{*} \ldots \varphi_{y_{k}}^{*}=\sum_{[b]} \Omega[b]
$$

can be expressed as a sum over configurations $[b]$ with positive weights $\Omega[b]$ that are calculable in polynomial time. In other words a solution to the bosonic sign problem in the $2 k$-point correlation function exists. Worldline representations are known to yield such solutions [11, 12].

In all cases where fermion sign problems have been solved, some transparent pairing mechanism exists. It may be hidden in the original formulation and require a new formulation to make the pairing transparent. When the pairing is unclear, sign problems remain unsoved [8]. In the future it is important to focus on problems without an obvious pairing mechanism.

\section{Acknowledgments}

I would like to thank A. Li for his collaboration and many helpful discussions. This work was supported in part by the Department of Energy grant DE-FG02-05ER41368.

\section{References}

[1] S. Christofi And C. Strouthos,

Three dimensional four-fermion models: A Monte Carlo study,

JHEP 0705, 088 (2007). 
[2] P. Gerhold And K. Jansen,

The Phase structure of a chirally invariant lattice Higgs-Yukawa model - numerical simulations, JHEP 0710, 001 (2007).

[3] S. Chandrasekharan, A new computational approach to lattice quantum field theories, Proceedings of Science (Lattice 2008), 003 (2008).

[4] S. ChandRaSEKhaRAN, Fermion bag approach to lattice field theories, Phys. Rev. D 82, 025007 (2010).

[5] S. Chandrasekharan AND A. LI, Fermion bag solutions to some sign problems in four-fermion field theories, Phys. Rev. D 85, 091502 (2012).

[6] S. ChANDRASEKHARAN, Solutions to sign problems in lattice Yukawa models, Phys. Rev. D 86, 021701(R) (2012).

[7] S. Catterall, R. Galvez, J. Hubisz, D. Mehta and A. Veernala, Non-abelian gauged NJL models on the lattice, Phys. Rev. D 86, 034502 (2012).

[8] S. ChandraseKharan and A. LI, The generalized fermion-bag approach, Proceedings of Science (Lattice 2011), 058 (2011).

[9] S. ChANDRASEKhARAN and A. LI, Fermion Bags, Duality, and the Three Dimensional Massless Lattice Thirring Model, Phys. Rev. Lett. 108, 140404 (2012).

[10] S. ChANDRASEKHARAN and A. LI, Fermion bag approach to the sign problem in strongly coupled lattice QED with Wilson fermions, JHEP 1101, 018 (2011).

[11] U. WOLFF, Simulating the All-Order Strong Coupling Expansion III: $O(N)$ sigma/loop models, Nucl. Phys. B 824, 254 (2010). Erratum-ibid. 834, 395 (2010).

[12] Y. D. Mercado and C. Gattringer, Monte Carlo simulation of the $S U(3)$ spin model with chemical potential in a flux representation, Nucl. Phys. B 862, 737 (2012). 